\title{
Interaction between von Willebrand factor and glycoprotein Ib activates Src kinase in human platelets : role of phosphoinositide 3 -kinase
}

\author{
Yi WU*, Naoki ASAZUMA*
}

Key word : Platelets, GPIb, Src, PI 3-kinase

Platelet adhesion to subendothelial structures is an early critical event in haemostasis and thrombosis. von Willebrand factor (VWF) is a major adhesive glycoprotein (GP) required for normal haemostasis under the condition of high shear stress, such as occurs in small arterioles and arterial capillaries ${ }^{1}$. In the presence of high shear stress or modulators such as botrocetin or ristocetin, VWF binds to the platelet membrane GPIbIX-V complex and initiates intracellular signals leading to platelet activation ${ }^{2}$. These include protein tyrosine phosphorylation, activation of protein kinase $\mathrm{C}$, activation of phosphoinositide 3-kinase (PI 3-kinase), elevation of the intracellular calcium ion concentration, and synthesis of thromboxane A $2^{3) \sim 8)}$. These intracellular signaling events play an essential role in the reorganization of platelet cytoskeletal actin filaments leading to platelet adhesion and spreading, and activation of integrin $\alpha_{\mathrm{II}} \beta_{3}$, with resultant platelet aggregation ${ }^{9) \sim 11)}$.

Recently, the events related to protein- tyrosine phosphorylation have emerged as important signals mediated by $\mathrm{GPIb}^{3 / \sim 612) \sim 14)}$. GPIb-mediated platelet activation in response to VWF plus botrocetin, shear stress or VWF from von Willebrand's disease type IIb patients induces tyrosine phosphorylation of multiple proteins, suggesting that vWF binding to GPIb causes the activation of tyrosine kinases, such as Syk and $\mathrm{Src}^{5}$. The proteins containing immunoreceptor tyrosine-based activation motif (ITAM), FcR $\gamma$-chain and Fc $\gamma$ RIIA, are involved for GPIb-mediated platelet activation processes, and tyrosine phosphorylation of Syk, linker for activation of T-cells (LAT) and phospholipase $\mathrm{C}_{\gamma} 2$ ( $\left.\mathrm{PLC}_{\gamma} 2\right)$ is dependent on FcR $\gamma$-chain ${ }^{6) 12 \sim 14)}$. Since these ITAMcontaining proteins lie downstream of activation of Src family kinases, it is important to investigate how GPIb stimulation mediates Src kinase activation. However, up to date, it has remained elusive how the GPIb-VWF interaction is coupled to activation of Src kinases.

Department of Clinical and Laboratory Medicine, University of Yamanashi, Tamaho, Nakakoma, Yamanashi 409-3898, Japan.

* These two authors contributed equally to this work. 
The GPIb-IX-V complex is composed of four different polypeptides, GPIb $\alpha, \operatorname{GPIb} \beta$, GPIX, and GPV, at a ratio of $2: 2: 2: 1^{2)}$. This complex is neither associated with GTPbinding proteins, nor possesses intrinsic tyrosine kinase activity. It also has no tyrosine residues to be phosphorylated. Recent studies have identified several signaling molecules associated with the cytoplasmic domain of GPIb-IX-V that play a critical role for signal transduction. One of these molecules is PI 3-kinase ${ }^{7) 15}$. The association of PI 3-kinase with GPIb-IX-V complex is mediated by the interaction between its $\mathrm{p} 85$ subunit and 14-3-3. PI 3-kinase plays multiple roles in functions of mammalian cells. Most of its effects depend on its activation with resultant production of D-3 lipids, such can be seen with Btk and PLC $\gamma^{16) \sim 18)}$. On the other hand, there are increasing lines of evidence indicating that the regulatory subunit of PI 3-kinase, $\mathrm{p} 85$, binds a diverse number of intracellular signaling proteins, especially Src family kinases ${ }^{19) \sim 24)}$, and regulates various cellular functions independent of the catalytic activity of PI 3-kinase ${ }^{25) 26}$. In this study, we sought to determine whether PI 3-kinase is involved in the GPIb-related signal transduction pathway.

With a number of receptors, especially those for growth factors and adhesive molecules, clustering of receptors is a prerequisite for signal transduction; simple binding of ligand does not suffice. It is widely accepted that the giant molecule of VWF, with its multimeric binding sites, induces clustering of GPIb molecules on the platelet membrane, thereby eliciting downstream signals. In accord with this notion, dispase-treated VWF or monomeric A 1 domains of VWF do not induce platelet aggregation ${ }^{2728)}$. However, there are several reports suggesting that simple ligand binding of GPIb elicits activation signals to some extent ${ }^{29) 30}$. In this report, we also sought to address this issue by using the recombinant A 1 domain, and cytochalasin D, an inhibitor of actin polymerization.

\section{Stimulation-dependent association of Src and Lyn with GPIb in platelets :}

The findings presented in this study demonstrate for the first time that Src kinases physically associate with GPIb and that they are activated by the interaction between GPIb and VWF. In a previous report, we found that an unidentified tyrosine kinase activity coprecipitated with GPIb upon VWFGPIb interaction. In the present study, by using Complete ${ }^{\mathrm{TM}}$, a mixture of protease inhibitors with a activity towards a wide range of proteases, we found that Src and Lyn dynamically associate with GPIb upon VWFGPIb interaction. Consistent with the increased level of GPIb-associated Src, the inclusion of Complete ${ }^{\mathrm{TM}}$ also allowed us to observe in immunoprecipitation kinase assays the pronounced autophosphorylation of a $60 \mathrm{kDa}$ protein associated with GPIb, as well as the included enolase substrate, implying that Src is the main candidate responsible for GPIb-associated tyrosine kinase activity.

\section{GPIb selectively stimulates activation of Src and its redistribution to the cytoskeleton :}

Previous studies have shown that cluster- 
ing of GPIb receptor by multimeric VWF induces actin polymerization ${ }^{10)}$, and stimulates Src and Lyn translocation to reorganized cytoskeleton at an early stage of platelet activation ${ }^{531)}$. As found in this study, translocation of Src kinases was inhibited by Cyto $\mathrm{D}$ pretreatment. This procedure allowed us to observe an increase in Src autophosphorylation and its kinase activity. Thus, rapid redistribution of Src kinases appears to have resulted in an underestimate of the increased activity of Src in the Triton-soluble fraction. These findings also imply that GPIbstimulated Src activation is not dependent on receptor clustering. This hypothesis is at odds with a widely held notion that GPIb clustering is a prerequisite for its downstream signaling. To address this issue, we used recombinant A 1 domain, instead of intact VWF, to stimulate platelets, since the monomeric A 1 domain is not able to cluster GPIb with subsequent reorganization of actin filaments. With platelets stimulated by A 1 domain plus botrocetin, Src, rather than Lyn, is selectively activated in a time-dependent manner. Moreover, A 1 domain plus botrocetin also stimulates Src association with GPIb, demonstrating that binding of A 1 domain to GPIb is indeed sufficient to activate $\mathrm{Src}$ without requirement of receptor clustering. Our findings also suggest that GPIb-stimulated Src activation is independent of outside-in integrin $\alpha_{\mathrm{II}} \beta_{3}$ signaling, since A 1 domain does not bind integrin $\alpha_{\mathrm{II}} \beta_{3}$. To the best of our knowledge, this is the first report showing that the interaction between GPIb and VWF specifically activates Src without receptor clustering. Given that PP 1, a specific inhibitor of Src family kinases, completely blocks tyrosine phos- phorylation of Syk, LAT, PLC $\gamma 2$ and ITAMcontaining proteins, $\mathrm{FcR} \gamma$-chain and Fc $\gamma$ RIIA, ${ }^{612) 13)}$, it is suggested that Src activation constitutes the main pathway for GPIb-mediated tyrosine phosphorylation. In contrast, Lyn activation was not observed upon GPIb stimulation, especially at the early stage, implying that Lyn has little, if any, functional role in GPIb signaling.

\section{Interaction of VWF with GPIb stimulates complex formation between Src, PI 3-kinase and GPIb :}

The constituents of GPIb-IX-V complex lack tyrosine-phosphorylated residues and other special binding motifs, such as prolinerich domain and SH 2 domain, to ensure the binding of Src to GPIb. Thus, it is likely that the association of Src with GPIb is not direct, mediated by another signaling molecule. A potential role for PI 3-kinase in GPIb signaling has been suggested by the observation that VWF binding to GPIb can induce the cytoskeletal association and activation of p 85/p 110 form of PI 3-kinase ${ }^{7}$. More interestingly, PI 3-kinase has been shown to associate with $\mathrm{GPIb}^{15}$. In this study, we detected a heterotrimeric complex of Src, PI 3-kinase and GPIb in lysates of platelets activated by VWF and botrocetin, which suggests that PI 3-kinase links Src with GPIb. Upon GPIb stimulation, the complex of GPIb, PI 3-kinase and Src appears to translocate to cytoskeleton, which is supported by the observation that Src and GPIb were recovered in $\mathrm{p} 85$ immunoprecipitates in RIPA-extract of cytoskeletal proteins. We then asked what could be the mode of interaction between 
GPIb, PI 3-kinase and Src. Upon GPIb stimulation, PI 3-kinase recovered in GPIb immunoprecipitates decreased in amount. Correspondingly, GPIb recovered in PI 3-kinase immunoprecipitates was also reduced, suggesting that these two molecules transolocate to cytoskeleton in a complex. When their translocation to cytoskeleton was blocked by Cyto D pretreatment, the level of their association remained constant, irrespective of stimulation, which implies that GPIb and PI 3kinase constitutively associate and their association remains constant even after platelet activation. On the other hand, Src association with GPIb or PI 3-kinase is dynamically dependent upon GPIb stimulation, especially in Cyto D-pretreated platelets. These findings taken together suggest that $\mathrm{Src}$ is recruited to the already-existing complex of GPIb and PI 3-kinase upon platelet activation. We then sought to determine which molecule, GPIb or PI 3-kinase, directly binds Src. Using an immunodepletion method with anti-p 85/PI 3kinase $\mathrm{Ab}$, we found that PI 3-kinase and $\mathrm{Src}$ always coexist in GPIb immunoprecipitates, which suggests that Src binds to PI 3-kinase, but not to GPIb.

\section{PI 3-kinase serves as adaptor protein to recruit Src to GPIb :}

PI 3-kinase plays a central role in the regulation of multiple cellular events, such as cell growth, vesicular trafficking, cytoskeletal organization, proliferation and apoptosis ${ }^{32 \sim \sim 34}$. Especially, a number of tyrosine kinases appear to be regulated by PI 3-kinase, by its production of D-3 lipids that act to recuit proteins to cell membranes. In this study, wortmannin had no effects on
GPIb-mediated Src activation, the formation of GPIb-Src-PI 3-kinase complex, or tyrosine phosphorylation of $\mathrm{FcR} \gamma$-chain, Syk, and $\mathrm{PLC}_{\gamma} 2$. Thus, the enzymatic activity of PI 3-kinase appears not to be required in this setting. It is known that $\mathrm{p} 85$ subunit of PI 3-kinase has several motifs that recruit signaling proteins. In some cells, it serves as an adaptor protein, independent of PI 3-kinase enzymatic activity, to regulate cell function. The association between Src and p 85 may theoretically be mediated by two distinct molecular interactions: one between the phosphotyrosine residues on $\mathrm{p} 85$ and the Src-SH 2 domain, and the second between the p 85 proline-rich sequences and the Src-SH 3 domain. In this study we found that $\mathrm{p} 85$ was not tyrosine-phosphorylated upon VWFbotrocetin stimulation. Using GST-Src-SH 3 pull-down assay, we found $\mathrm{Src}$ association with the GPIb-PI 3-kinase complex is mediated by the interaction between Src-SH 3 domain and the proline-rich motif of $\mathrm{p} 85$. Thus, the present data suggest that $\mathrm{p} 85$ serves as a scaffolding protein to recruit Src to GPIb. Indeed, association of Src with $\mathrm{p} 85$ subunit of PI 3-kinase via its SH 3 domain is a common mode of cross-talking, and contributes to intracellular targeting of $\mathrm{Src}$ in other cells ${ }^{32) 33}$. Based on these findings, we suggest that PI 3-kinase constitutively associates with GPIb, and that ligand binding of GPIb mediates certain conformational change of the GPIb-p 85/PI 3-kinase complex, which recruits Src. Previous studies have shown that the association of PI 3-kinase with GPIb is mediated by $14-3-3$, which plays a critical role in GPIb-dependent activation of integrin $\alpha_{\mathrm{III}} \beta_{3}{ }^{355}$. Whether p 85/PI 3-kinase-dependent association of Src with GPIb may also be 
regulated by 14-3-3 awaits to be elucidated.

In conclusions, our study demonstrates for the first time that the vWF-GPIb interaction stimulates Src association with GPIb, and Src activation. GPIb-associated p 85 subunit of PI 3-kinase functions as a scaffording protein, recruiting Src to GPIb, thereby leading to its activation. The physical interaction of Src with GPIb and its early activation imply that Src activation constitutes the paramount pathway of GPIb-related tyrosine phosphorylation events.

\section{References}

1) Ruggeri ZM. von Willebrand factor. J Clin Invest 99 : 559-564, 1997.

2) Clemetson KJ. Platelet glycoprotein Ib-IX-V complex. Thromb Haemost $78:$ 266-270, 1997.

3) Razdan K, Hellums JD, Kroll MH. Shear-stressinduced von Willebrand factor binding to platelets causes the activation of tyrosine kinase (s). Biochem J 302: 681-686, 1994.

4) Ozaki Y, Satoh K, Yatomi Y, Miura S, Fujimura Y, Kume S. Protein tyrosine phosphorylation in human platelets induced by interaction between glycoprotein $\mathrm{Ib}$ and von Willebrand factor. Biochim Biophys Acta 1243: 482-488, 1995.

5) Asazuma N, Ozaki Y, Satoh K, et al. Glycoprotein Ib-von Willebrand factor interactions activate tyrosine kinases in human platelets. Blood 90 : 47894798, 1997.

6) Canobbio I, Bertoni A, Lova P, et al. Platelet activation by von Willebrand factor requires coordinated signaling through thromboxane A 2 and $\mathrm{Fc} \gamma$ IIA receptor. J Biol Chem 276 : 26022-26029, 2001.

7) Jackson SP, Schoenwaelder SM, Yuan Y, Rabinowitz I, Salem HH, Mitchell CA. Adhesion receptor activation of phosphatidylinositol 3-kinase : von Willebrand factor stimulates the cytoskeletal association and activation of phosphatidylinositol 3-kinase and pp 60 c-src in human platelets. J Biol Chem 269 : 27093-27099, 1994.

8) Kroll MH, Harris TS, Moake JL, Handin RI, Schafer AI. von Willebrand factor binding to platelet GPIb initiates signals for platelet activation. J Clin Invest $88: 1568-1573,1991$.

9) Yap CL, Hughan SC, Cranmer SL, et al. Synergistic adhesive interactions and signaling mechanisms operating between platelet glycoprotein Ib/IX and integrin $\alpha \operatorname{IIb} \beta 3$. Studies in human platelets ans transfected Chinese hamster ovary cells. J Biol Chem 275 : 41377-41388, 2000.

10) Yuan $Y$, Kulkarni S, Ulsemer $P$, et al. The von Willebrand factor-glycoprotein $\mathrm{Ib} / \mathrm{V} / \mathrm{IX}$ interaction induces actin polymerization and cytoskeletal reorganization in rolling platelets and glycoprotein $\mathrm{Ib} / \mathrm{V} /$ IX-transfected cells. J Biol Chem 274:36241-36251, 1999.

11) Zaffran Y, Meyer SC, Negrescu E, Reddy KB, Fox JE. Signaling across the platelet adhesion receptor glycoprotein Ib-IX induces $\alpha \operatorname{IIb} \beta 3$ activation both in platelets and a transfected Chinese hamster ovary cell system. J Biol Chem 275 : 16779-16787, 2000.

12) $\mathrm{Wu} \mathrm{Y}$, Suzuki-Inoue K, Satoh K, et al. Role of Fc receptor $\gamma$-chain in platelet glycoprotein Ib-mediated signaling. Blood 97 : 3836-3845, 2001.

13) Falati S, Edmead CE, Poole AW. Glycoprotein Ib-VIX, a receptor for von Willebrand factor, couples physically and functionally to the Fc Receptor $\gamma$ chain, Fyn, and Lyn to activate human platelets. Blood 94 : 1648-1656, 1999.

14) Berndt MC, Shen Y, Dopheide SM, Gardiner EE, Andrews RK. The vascular biology of the glycoprotein Ib-IX-V complex. Thromb Haemost $86: 178$ 188, 2001.

15) Munday AD, Berndt MC, Mitchell CA. Phosphoinositide 3-kinase forms a complex with platelet membrane glycoprotein Ib-IX-V complex and 14-3-3 $\xi$. Blood 96 : 577-584, 2000.

16) Li Z, Wahl MI, Eguinoa A, Stephens LR, Hawkins PT, Witte ON. Phosphatidylinositol 3-kinase-gamma activates Bruton's tyrosine kinase in concert with Src family kinases. Proc Natl Acad Sci USA 94:1382013825, 1997.

17) Falasca M, Logan SK, Lehto VP, Baccante G, Lemmon MA, Schlessinger J. Activation of phospholipase $\mathrm{C} \gamma$ by PI 3-kinase-induced $\mathrm{PH}$ domain-mediated membrane targeting. EMBO J 17 : 414-422, 1998.

18) Bobe R, Wilde JI, Maschberger P, et al. Phosphatidylinositol 3-kinase-dependent translocation of phospholipase $\mathrm{C}_{\gamma} 2$ in mouse megakaryocytes is independent of Bruton's tyrosine kinase translocation. Blood 97 : 678-684, 2001.

19) Fincham VJ, Brunton VG, Frame MC. The SH 3 domain directs acto-myosin-dependent targeting of $\mathrm{v}$-Src to focal adhesions via phosphatidylinositol 3kinase. Mol Cell Biol 20 : 6518-6536, 2000.

20) Grey A, Chen Y, Paliwal I, Carlberg K, Insogna K. Evidence for a functional association between phosphatidylinositol 3-kinase and c-src in the spreading response of osteoclasts to colony-stimulating factor-1. Endocrinology $141: 2129-2138,2000$.

21) Mak $P$, He $Z$, Kurosaki $T$. Identification of amino acid residues required for a specific interaction between Src-tyrosine kinase and proline-rich region of phosphatidylinositol 3-kinase. FEBS Lett 397 : 183-185, 1996.

22) Prasad KV, Janssen O, Kapeller R, Raab M, Cantley LC, Rudd CE. Src-homology 3 domain of protein kinase p 59 fyn mediates binding to phosphatidylinositol 3-kinase in T cells. Proc Natl Acad Sci USA 90 : 7366-7370, 1993.

23) Renzoni DA, Pugh DJ, Siligardi G, et al. Structural and thermodynamic characterization of the interaction of the SH 3 domain from Fyn with the prolinerich binding site on the p 85 subunit of PI 3-kinase. Biochemistry 35 : 15646-15653, 1996.

24) Rittenhouse SE. Phosphoinositide 3-kinase activation 
and platelet function. Blood 88: 4401-4414, 1996.

25) Jascur T, Gilman J, Mustelin T. Involvement of Phosphatidylinositol 3-kinase in NFAT activation in $\mathrm{T}$ cells. J Bio Chem 272 : 14483-14488, 1997.

26) Kang H, Schneider H, Rudd CE. Phosphatidylinositol 3-kinase p 85 adaptor function in T-cells. J Bio Chem $277: 912-921,2002$.

27) Miura S, Fujimura Y, Sugimoto M, et al. Structural elements influencing von Willebrand factor (VWF) binding affinity for platelet glycoprotein Ib within a dispase-digested vWF fragment. Blood 84 : 1553-1558, 1994.

28) Cruz MA, Handin RI, Wise RJ. The interaction of the von Willebrand factor-A 1 domain with platelet glycoprotein Ib/IX. The role of glycosylation and disulfide bonding in a monomeric recombinant A 1 domain protein. J Bio Chem 268 : 21238-21245, 1993.

29) Yanabu M, Ozaki Y, Nomura S, et al. Tyrosine phosphorylation and $\mathrm{p} 72^{\mathrm{syk}}$ activation by an anti-GPIb monoclonal antibody. Blood 89 : 1590-1598, 1997.

30) Satoh K, Asazuma N, Yatomi Y, et al. Activation of protein-tyrosine kinase pathways in human platelets stimulated with the A 1 domain of von Willebrand factor. Platelets $11: 171-176,2000$.

31) Yuan Y, Dopheide SM, Ivanidis C, Salem HH, Jackson SP. Calpain regulation of cytoskeletal signaling complexes in von Willebrand factor-stimulated platelets. Distinct roles for glycoprotein Ib-V-IX and glycoprotein IIb-IIIa (integrin $\alpha \operatorname{IIb} \beta 3$ ) in von Willebrand factor-induced signal transduction. J Biol Chem 272 : 21847-21854, 1997.

32) Wymann MP, Pirola L. Structure and function of phosphoinositide 3-kinases. Biochim Biophys Acta 1436 : 127-150, 1998.

33) Geltz NR, Augustine JA. The p 85 and p 110 subunits of Phosphatidylinositol 3-kinase are substrates, in vitro, for a constitutively associated protein tyrosine kinase in platelets. Blood 91 : 930-939, 1998.

34) Fruman DA, Meyers RE, Cantley LC. Phosphoinositide kinases. Annu Rev Biochem 67 : 481-507, 1998.

35) Gu M, Xi X, Englund GD, Berndt MC, Du X. Analysis of the roles of 14-3-3 in the platelet glycoprotein Ib-IXmediated activation of integrin $\alpha \operatorname{IIb} \beta 3$ using a reconstituted mammalian cell expression model. J Cell Biol 147 : 1085-1096, 1999. 